\title{
An Automated Hydraulic Optimization Approach for RCP Impeller Using MOGA
}

\author{
Xide Lai*, Daoxing Ye, Xiang Zhang, Qiuqin Gou and Xiaoming Chen \\ School of Energy and Power Engineering, Xihua University, Chengdu, (610039), China \\ ${ }^{*}$ Corresponding author
}

\begin{abstract}
This paper presents an automated hydraulic optimization approach for impeller of a reactor coolant pump (RCP) combining a blade geometric modeller and parametric generator with automatic CFD solution procedure and multiobjective genetic algorithm. The approach depends on the cooperation between a genetic algorithm, a database and user defined objective functions and constraints which comprises hydraulic performances, structural and geometric constraint functions. In order to demonstrate the performance of the presented approach A mixed-flow RCP impeller was optimized.
\end{abstract}

Keywords-optimization; RCP; impeller; MOGA

\section{INTRODUCTION}

Although CAD/CFD software has widely used in design of a pump, it is still a great challenging task to optimize the hydraulic performance of a pump by trial and error modifying its component geometries, because it not only requires an advanced optimization software, but also the key components of the system employed within the optimization procedure should ensure the optimization loop to work robustly, accurately and efficiently [1-3]. The most difficult task is how to automatically optimize the performance of impeller's rotating blade system which consist of a number of blades. The blade shape of a mixed-flow RCP impeller usually has sculpture surfaces feature to meet the requirements of hydraulic performances. Hydraulic optimization design for blades is an intricate task involving a number of different objectives and constraints coming from hydraulics, structure and geometry [1-4]. Although designers have already applied CFD software to assess their hydraulic designs, to more tightly integrate CFD analyses is further required in the design procedure by using efficient optimization methods to get more reliable design [2-7]. An automated hydraulic optimization approach which combines a blade geometry parametrization generator with an automatic CFD solution process and an optimization algorithm that are suitable for hydraulic optimization of a mixed-flow pump impeller is presented in this paper.

\section{OPTIMIZATION PROCEDURE}

The basic idea of the presented approach is to speed the new blade system design on the basis of the knowledge acquired from previous designs of similar pump. As shown in Fig.1, this hydraulic optimization toolchain mainly comprises essentially of a parametric blade geometry modeler, a 3D flow simulation software including parametric mesh generation tool, a coupled CFD solver and post-processor for turbomachinery, and an efficient optimizer with multi-objective genetic algorithm (MOGA).



FIGURE I. HYDRAULIC OPTIMIZATION LOOP FOR PUMP IMPELLER'S BLADES SYSTEM

The initial blade model can be obtained from the new blade design with the hydraulic, geometrical and mechanical requirements imposed by the user by means of the existed blade design tool such as CFturbo ${ }^{\circledR}$, or from the previous designs of the similar blade. The blade geometry is firstly required to be parameterized with a reliable parametric blade modeler. The second step is to construct the approximate relation between the parametric geometry and hydraulic boundary conditions, and the hydraulic performance as well. An artificial neural network (ANN) contains free parameters that have to be adapted in order to fit the database samples through "learning process". After the mapping of the database samples, the ANN can predict the hydraulic performance of blade geometries under given flow boundary conditions that are not inside the database. To implement prediction of hydraulic performance for blade systems, a speed and robustness the CFD package consisted of mesh generator, flow solver and post-processor is plugged into an optimization loop for flow simulation. The third step is to seek a new optimized geometry to be analyzed by the $3 \mathrm{D}$ flow simulation. It is executed by using an optimization procedure of genetic algorithm, the hydraulic performance of blade system being evaluated by means of the trained neural network. The global performance of blade system is evaluated through singleobjective or multi-objective functions. In the fourth step, the newly designed blade geometry provided by the optimizer is evaluated by ways of the flow simulation and this new sample is added to the database. Finally, the evaluated hydraulic performance is compared to the target. If this new blade geometry has not been achieved the target performance, another design iteration is started, and the same process is 
repeated until the new blade geometry meet the target performance is obtained. A new iteration always starts with the neural network learning. Along with the optimization design proceeds, the database grows, the approximate relation can further improved and therefore can find a better localization of the real optimum.

\section{Optimization Problems AND Algorithms}

\section{A. Optimization Problems for Hydraulic Design of RCP Impeller}

For hydraulic design optimization problems of a pump, a several of objectives (such as hydraulic efficiency, cavitation, pressure pulsation, pressure distribution on blade, the required performance curve, increasing head or discharge, lowering the noise etc.) and/or constraints(such as structural stress, dimensions and manufacture requirements) and a large number of design variables may be concerned. The Multi-Objective Optimization problems of hydraulic machinery can be described as:

$$
\begin{aligned}
& \max \left\{z_{1}=f_{1}(x), z_{2}=f_{2}(x), \cdots, z_{q}=f_{q}(x)\right\} \\
& \text { s.t. } \quad g_{i}(x) \leq 0, \quad i=1,2, \cdots, m
\end{aligned}
$$

Where: $\quad f_{\mathrm{i}}(x)$ are objective functions, $x=\left(x_{1}, x_{2}, \cdots x_{n}\right) \in R^{n}$ are design variables, $g_{\mathrm{i}}(x)$ are constraint functions.

\section{B. Objective Functions and Constraints}

A good design of blade system must satisfy the hydraulic performance requirements as well as the mechanical and manufacturing constraints. There are different design objectives of the detailed hydraulic optimization in different cases of hydraulic machines [7].

\section{Optimization Algorithms}

As many local optima may exist in the design space during blade optimizing process and a global optimization technique with a fast and robustness numerical optimization algorithm is accordingly required [3,5]. The GAs can obtain the global optimum without being trapped into local optima in MultiObjective Optimization (MOO) problems and also accommodate any ready-to-use analysis/evaluation software as a black-box tool, such as the CFD codes used in the applications without requiring access to its source code [6]. In fact, it is the only necessary condition that there is the availability of an appropriate evaluation software as a blackbox tool by the GA and design variables and well-defined objective functions for carrying out a GA-based optimization [7].

\section{HydRAulic Optimization FOR A MiXED-FLOW RCP IMPELLER}

\section{A. Optimization of the Mixed-flow RCP Impeller}

Table 1 shows a mixed-flow RCP to be optimized and Fig. 2 shows the original RCP impeller. The initial hydraulic efficiency is $80 \%$ and expected is larger than $82 \%$ and head larger than $97 \mathrm{~m}$ at the best efficiency point. The design optimization of the RCP impeller is handled as a 4-objective optimization. The first objective (F1t) requires a maximum weighted efficiency for 5 different operating points. The second objective (F2t) is related to control the $\mathrm{H} \sim \mathrm{Q}$ curve (to keep stability at operation in system). The third objective (F3t) is to increase the weighted head for 5 different operating points without increasing the input power. The fourth objective (F4t) is regarding cavitation behaviour which corresponds to control static pressure distribution on the blade surfaces, to increase the minimum pressure of impeller inlet and lower pressure pulsation inside pump. This RCP will be operating at the inlet pressure of 15Mpa.

TABLE I. THE SPECIFICATIONS FOR A MIXED-FLOW RCP IMPELLER TO BE OPTIMIZED

\begin{tabular}{lllllll}
\hline Head(m) & $\begin{array}{l}\text { flow } \\
\text { rate(m/3) }\end{array}$ & $\begin{array}{l}\text { Speed } \\
(\mathbf{r} / \mathbf{m i n})\end{array}$ & $\begin{array}{l}\text { impeller outlet } \\
\text { Max.Dia.(mm) }\end{array}$ & $\begin{array}{l}\text { impeller inlet } \\
\text { Min.Dia.(mm) } \\
\text { Dia.(mm) }\end{array}$ & $\begin{array}{l}\text { N.of } \\
\text { blade }\end{array}$ & $\begin{array}{l}\text { N.of } \\
\text { diffuser } \\
\text { vane }\end{array}$ \\
\hline 95 & 23800 & 1485 & 885 & 690 & $\begin{array}{l}\text { Target } \\
\text { eff. ( \%) }\end{array}$ \\
\hline
\end{tabular}

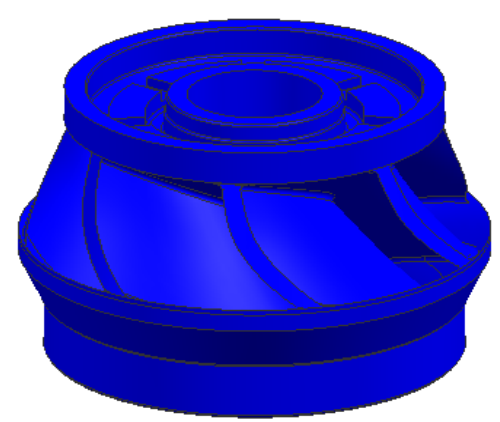

FIGURE II. RCP IMPELLER TO BE OPTIMIZED.

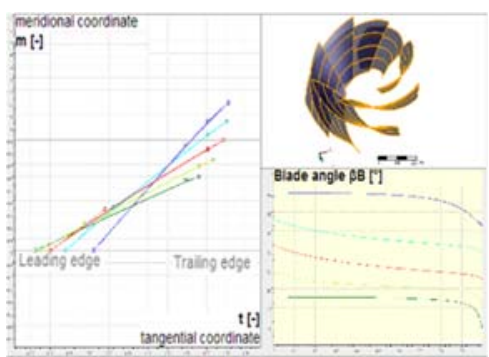

FIGURE III. BLADE GEOMETRIC PARAMETERIZATION. 


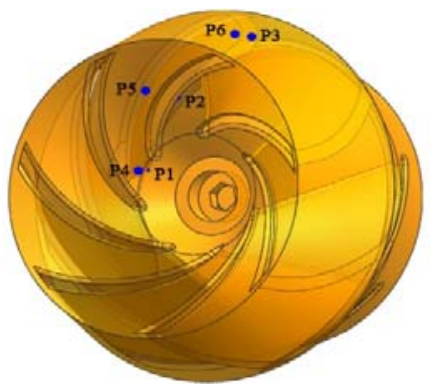

FIGURE IV. RCP IMPELLER'S FLOW DOMAIN ANDMONITORING POINTS OF PRESSURE

\section{B. Blade Geometric Parameterization and Design Variables}

The geometric parametrization is a key element in the success of the automatic hydraulic optimization for blade system. It is idealized that the parametrization of the blade geometry should be able to provide flexible variation of the blade geometric shape with as few design variables as possible. Pump designers are used to do design with 2D sections on stream surface that are then stacked to the 3-dimensional blade geometry [6-7]. How to parametrize a blade for optimization had been presented in ref.[7]. As shown in Fig.3, 5 streamline profiles on blade were taken in this case.

After blade geometric parameterization, the blade shape can be lofted by the 5 streamline profile's curves on streamsurfaces and a parameterized thickness distribution onto the parameterized mean camber curves with the parametric blade modeller. The parametric setup employs geometries of blade profiles on each defined stream surfaces during geometric parametrization, design variables are LE and TE angles, set angles and LE radius for the blade profiles on each defined stream surfaces, and the number of blade. In the case of RCP impeller, the design variables are:

$$
x=\left\{\beta_{i 1}, \gamma_{i}, R_{L E i}, \beta_{i 1}, \mathrm{~N}\right\} \quad i=1,2,3,4,5
$$

Where: $\gamma$ is set angle of profile on stream surface, $\beta_{1}$ is Leading edge set angle, $\beta_{2}$ is Trailing edge set angle, $R_{\mathrm{LE}}$ is radius of Leading edge, $i$ is the number of stream surfaces, $N$ is the numbers of blade.

\section{Objective Functions and Constraints}

In the optimization of mixed-flow RCP, the multi-objective functions can be defined as:

$$
\max \{\eta(x), H(x)\} ; \min T_{r}(x) ; \max \left\{p_{\text {in }}(x)\right\}
$$

Where: $\eta(x)$ is hydraulic efficiency; $H(x)$ is the head; $T_{\mathrm{r}}(x)$ is input torque; $P_{\text {in }}(x)$ is average of inlet static pressure;

5 section profiles on stream surfaces are taken to control the shape of a blade during blade parameterization, so there are totally 21 design variables, and constraints of design variables for the impeller blade are:

$$
\text { s.t. }\left\{\begin{array}{l}
19^{\circ} \leq \beta_{i 1} \leq 43^{\circ} \\
12^{\circ} \leq \beta_{i 2} \leq 35^{\circ} \\
9.0 \leq R_{L E_{-} i} \leq 11.5 \\
50^{\circ} \leq \gamma_{i} \leq 72^{\circ} \\
6 \leq N \leq 8
\end{array}\right.
$$

\section{Multi-objective and Multi-operating Points' Optimization}

To solve the optimization problem as the described above, we employ the optimization procedure described in section 2 for Multi-objective and Multi-operation point hydraulic optimization. During optimization, the integral flow passage combining the impeller with the same diffuser vanes is employed to perform flow simulation. The mesh for flow simulation during optimization was generated with the grid generation tool matching the parametric setup of the blade geometry modeller, so that the grid can be easily aligned with the geometry. The parametric grid generator enables the use of the same mesh topology and refinement for all simulated cases.

As MOGA is employed in hydraulic optimization of blade system, a database can be generated with discrete levels to keep the global of samples, 40 samples are generated and stored in the database. Each sample includes the hydraulic performance, 21 design variables and flow data. During GA implement, Initial population was set up to 100, parent population of loop was set up to 50 and samples be set up 40, and the max iteration steps assumed as 40 for automatic optimization.

\section{E. Optimization Results and Analysis}

Fig. 5 shows the performance curves (efficiency, head and input power vs. flowrates) of the before and after optimization. The efficiency has up to $83.1 \%$ at BEP, and the head is $96.6 \mathrm{~m}$ at the design point.

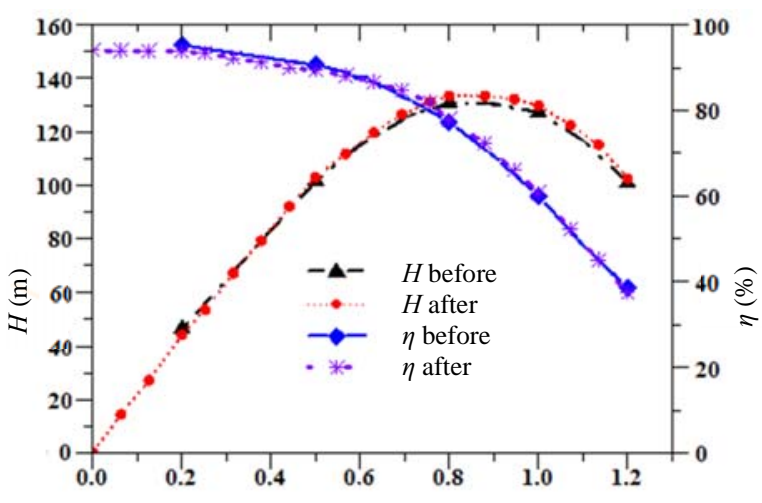

Flow rate

FIGURE V. PERFORMANCE CURVES OF THE BEFORE AND AFTER OPTIMIZATION 


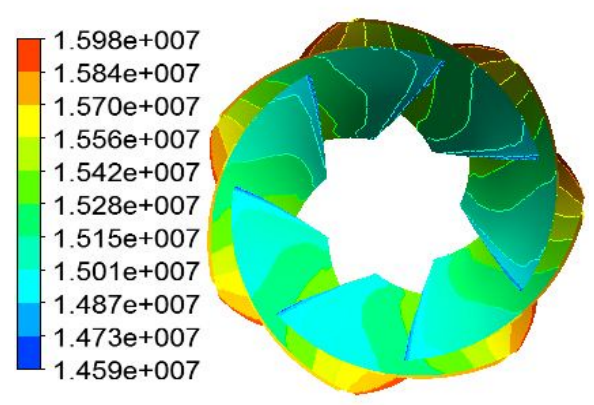

FIGURE VI. STATIC PRESSURE DISTRIBUTION AFTER OPTIMIZED AT DESIGN POINT

Fig.6 shows the static pressure distribution after optimization on blade surface at design operating point. It can be found that the optimized blade has great improvement in the static pressure distribution in wide operating range from $0.2-$ $1.2 Q_{\mathrm{d}}$.

In order to evaluate the safety caused by dynamic stress of blade, the pressure pulsation monitoring point was setup as shown in Fig.4, and numerically computed for the monitoring points P1-P6 as shown in Fig.7 at at design operating point as an example. It can be found that the optimized blade has greatly decreased in the amplitude of pressure pulsation in wide operating range.

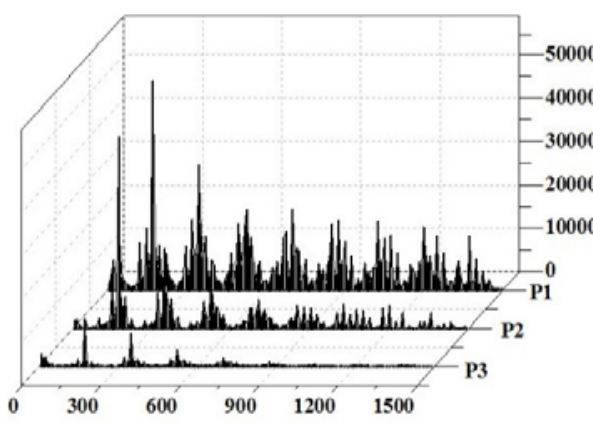

Frequncy $(\mathrm{Hz})$

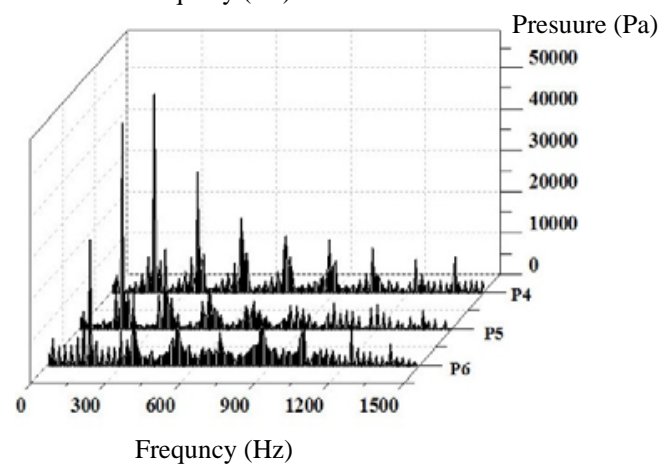

FIGURE VII. PRESSURE PULSATION FREQUENCY-DOMAIN DIAGRAM OF THE MONITORING POINTS INSIDE THE IMPELLER AT THE DESIGN POINT $\left(1.0 Q_{\mathrm{d}}\right)$

\section{CONCLUSION}

From the above results after optimization, it has shown that the automated hydraulic optimization approach for impeller presented in this paper can obtain more efficient design processes and greatly shorten design time schedule and get more reliable hydraulic design.

(1) To more easily establish automatic hydraulic optimization toolchain for hydraulic machine's blades system, the GA-based optimization is one of best choice. The existed or commercial software of the parametric blade geometry modeller, CFD solver including parametric mesh generation tool and post-processor for turbomachinery can be adopted to implement the relative requirements without requiring access to their source codes.

(2) During hydraulic optimization of the rotating blade system in pumps, it is recommended that the single-object optimization should be firstly employed to obtain a preoptimized model and then take this model as the input of multiobjective optimization. The Multi-Objective Optimization can improve and balance the complex hydraulic performances.

\section{ACKNOWLEDGMENTS}

The research was supported by the Major Research Plan of Sci.\&Tech. of Sichuan Province in China (Grant No.2017JY0047, 2017NZ0031, 2017GZ0053), and partially supported by the Foundation of Key Laboratory of Fluid and Power Machinery (Xihua University), Ministry of Education of China. Their supports are greatly appreciated.

\section{REFERENCES}

1] Xide Lai. Hydraulic optimization design of Hydro Turbine based on Performance prediction [J]. J. of Hydrodynamics,(Ser.A) 2002, （6） $1-8$ (in Chinese)

[2] R.Schilling, S.Thum, N.Muller, S.Kramer, N.Riedei, W.Moser. Design Oprimization of hydraulic machinery bladings by multilevel cfdtechinque[C]. Proceeding of the 21st IAHR Symposium on Hydraulic Machinery and System, 2002, Lausanne, Switzerland.

[3] Kyriacou, S.1 ; Kontoleontos, E.2; Weissenberger, S.2; Mangani, L. et al. Evolutionary algorithm based optimization of hydraulic machines utilizing a state-of-the-art block coupled CFD solver and parametric geometry and mesh generation tools[C]. Proceeding of 27th IAHR Symposium on Hydraulic Machinery and Systems, 2014, Montreal, Canada

[4] A Semenova, D Chirkov, A Lyutov, et al. Multi-objective shape optimization of runner blade for Kaplan turbine[C]. Proceeding of 27th IAHR Symposium on Hydraulic Machinery and Systems, 2014, Montreal, Canada

[5] S. Pierret, A. Demeulenaere, B. Gouverneur, Ch. Hirsch, "Designing turbomachinery blades with the function approximation concept and the navier-stokes equations", 8th AIAA/NASA/USAF/ISSMO Symposium on MDO, Sep6-Sep8, 2000,Long Beach, CA

[6] Georgia N. Koini, Sotirios S. Sarakinos, et al. A software tool for parametric design of turbomachinery blades [J]. Advances in Engineering Software, No. 40, pp:41-51,2009

[7] Xide Lai, Xiaoming Chen, Xiang Zhang. An Approach to automatically optimize the Hydraulic performance of Blade System for Hydraulic Machines using Multi-objective Genetic Algorithm[C], Proceeding of 28th IAHR Symposium on Hydraulic Machinery and Systems, Grenoble, France, July 2016 